\title{
Forcing and Dynamics of Seafloor-Water Column Exchange on a Broad Continental Shelf
}

\author{
BY WILLIAM B. SAVIDGE, ANN GARGETT, \\ RICHARD A. JAHNKE, JAMES R. NELSON, \\ DANA K. SAVIDGE, R. TIMOTHY SHORT, \\ AND GEORGE VOULGARIS
}

\begin{abstract}
Relict sediments of elevated permeability characterize the majority of continental shelves globally (Emery, 1968). In these settings, interactions between benthic boundary layer (BBL) flows and seabed topography generate pressure fluctuations that drive advective and dispersive porewater transport, dramatically increasing the magnitude and variability of porewater solute and particulate exchange across the sediment-water interface (Huettel et al., 1996; Huettel and Rusch, 2000). On broad shallow shelves with a relatively large area-to-volume ratio, the seafloor's role is magnified. Energetic events may reorganize bedforms across a significant fraction of the shelf, leading to altered exchange dynamics that may persist long after the organizing event. Ecosystem-based management of both resources and environmental status requires improved fundamental understanding of dynamic benthic exchange processes. Scattered, short-time-scale observations are unlikely to capture the full spectrum of events that affect sediment-water exchanges; a persistent observational presence on the seafloor is required.
\end{abstract}


The Benthic Observatory and Technology Testbed On the Mid ShelfUnderstanding Processes (BOTTOMSUP) project was designed to measure the seabed response to physical forces acting across a spectrum of spatial and temporal scales. The observatory site is located $65 \mathrm{~km}$ east of St. Catherine's Island, Georgia, at 27-m water depth on the broad southeastern US continental shelf. Regional (100-km) sea surface currents and wave fields are monitored by a shore-based Wellen Radar (WERA) high-frequency radar network. A five-beam vertical acoustic Doppler profiler (600-kHz VADCP) measures current velocities throughout the water column. Full water-column stratification is monitored using a thermistor chain. A seafloor-mounted rotating sonar generates images of bottom topography within a 6-m-radius circle at hourly intervals. These instruments are linked to the existing South Atlantic

William B. Savidge (william.savidge@ skio.usg.edu) is Assistant Research Professor, Skidaway Institute of Oceanography, Savannah, GA, USA.

Ann Gargett is Emerita Professor, Old Dominion University, Norfolk, VA, USA. Richard A. Jahnke is Professor, Skidaway Institute of Oceanography, Savannah, GA, USA. James R. Nelson is Associate Professor, Skidaway Institute of Oceanography, Savannah, GA, USA.

Dana K. Savidge is Assistant Professor, Skidaway Institute of Oceanography, Savannah, GA, USA. R. Timothy Short is Chemical Sensors Group Manager, Marine Technology Program, SRI International, Saint Petersburg, FL, USA. George Voulgaris is Professor, University of South Carolina, Columbia, SC, USA.
Bight Synoptic Offshore Observational Network observatory and transmit data to shore at hourly intervals. Additional observatory instrumentation provides high-temporal-resolution measurements of basic meteorological parameters, nondirectional wave heights, above- and within-water photosynthetically active radiation (PAR), and near-surface and near-bottom salinity, temperature, and chlorophyll fluorescence. Further information for all observing instruments can be obtained at http://www.skio.usg.edu/ Skioresearch/physical/. Together, these sensor systems provide a high-resolution record of forces and bottom topographic changes that control BBL, porewater, and sediment dynamics.

Within the context of these largerscale observations, BOTTOMS-UP has also deployed a set of stand-alone instruments on the seafloor to measure the responses to larger-scale forcing within the bottom boundary layer and sediment. Particle dynamics are monitored using a tripod equipped with two acoustic Doppler velocimeters (ADVs; $10 \mathrm{MHz}$ ) to measure water velocities at high resolution near the bed and an upward- and downward-looking acoustic backscatterance sensor (four frequencies each) to measure size-specific suspended particle concentrations (Voulgaris et al., 1998). A pair of fluorometer/turbidity sensors are coupled with the ADVs to produce eddy correlation sensors for suspended particulates. A second tripod holds an array of five shuttered fluorometers to sample the gradient of suspended materials in the bottom two meters of the boundary layer at six-minute intervals. Fluid exchange rates across the interface are estimated using a vertical thermistor array. High- temporal-resolution sampling $\left(1 \mathrm{~min}^{-1}\right)$ of the propagation of temperature fluctuations in the overlying water into the sediment are being used as a proxy for movement of water and solutes. Changes in the chemical environment within porewater in response to external forcing are measured with a vertical array of redox electrodes. An in situ membraneinlet mass spectrometer (Short et al., 2001; Bell et al., 2007) has been modified with a probe to sample vertical gradients of dissolved gases (primarily $\mathrm{O}_{2}, \mathrm{CO}_{2}$, $\mathrm{CH}_{4}, \mathrm{~N}_{2}$, and $\mathrm{Ar}$ ) within porewaters. The instrument has been deployed for one- to three-day intervals and has generated continuous gas profiles during those times.

\section{INITIAL OBSERVATIONS}

We focus here on initial observations from the sonar and VADCP instruments to demonstrate the dynamics of water column-sediment interactions in an open continental shelf setting. VADCP measurements suggest the occurrence of Langmuir supercells (LSCs) at the BOTTOMS-UP observatory site. In wind- and wave-driven surface waters, Langmuir circulations are parallel counterrotating vortices oriented roughly downwind, with maximum downwind velocities at the surface convergences between the vortex pairs. Under extended forcing, these circulations penetrate to the seafloor and provide a potentially important mechanism for vertical and horizontal transport of sediment resuspended in the thin, highly sheared bottom boundary layer associated with strong surface waves. Langmuir supercell circulation is schematically displayed at the top of Figure 1. Major expected features 
of this circulation relative to the central line of surface convergence are: (1) downwind velocity generally more intense near the seafloor and (2) downward vertical velocity and divergence in cross-wind velocities throughout most of the lower water column. These features are all readily observable in the VADCP data: vertical lines in Figure 1 highlight two examples, with arrows at the second occurrence, demonstrating the phase relations associated with
Langmuir circulation.

LSCs were first documented at the LEO-15 observatory (Gargett et al., 2004). They suggested that LSC events play a major role in sediment transport on the inner New Jersey shelf.

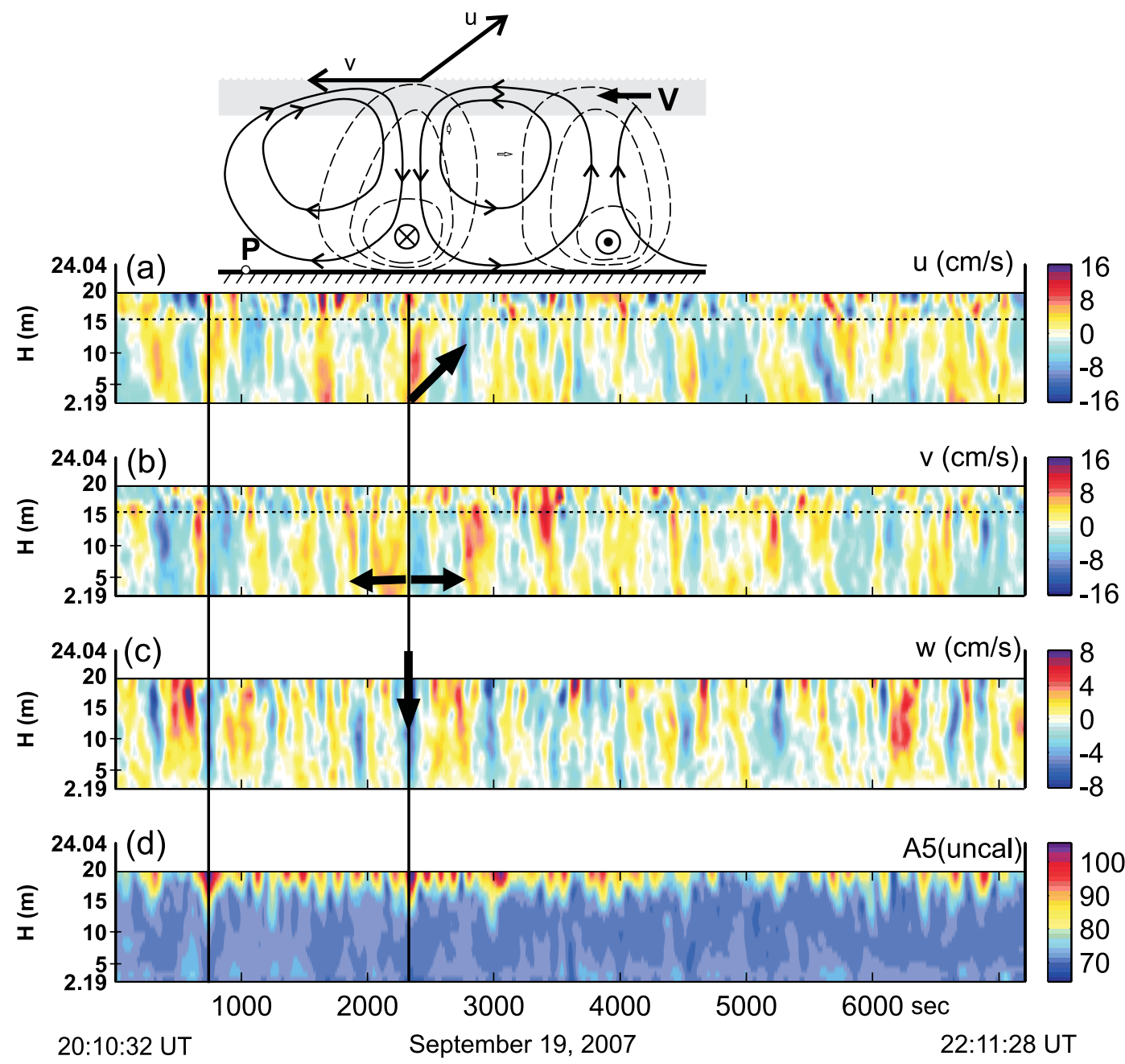

Figure 1. Three-dimensional fluctuation velocity field derived from two hours of VADCP data taken at the Benthic Observatory and Technology Testbed On the Mid Shelf-Understanding Processes (BOTTOMS-UP) observatory on the Georgia continental shelf during a storm. Colors code the fields of fluctuating (a) downwind (u), (b) crosswind (v), and (c) vertical ( $w$ ) velocities, and of vertical beam acoustic backscatter amplitude ( $d$, corrected for radial spreading and water attenuation, but not calibrated). Although the height above bottom $(\mathrm{H})$ of the mean water surface at the time of this record is $27 \mathrm{~m}$, large wave heights make the water column above $\mathrm{H}=20 \mathrm{~m}$ inaccessible to continuous observation. Horizontal lines in (a) and (b) denote the height above which horizontal velocities calculated from slanted acoustic beams may be contaminated by sidelobe effects. The cartoon at top shows the crosswind spatial structure of Langmuir supercells (Gargett et al., 2004) in the relevant coordinate system, with $\mathrm{x}=$ downwind direction. Downwelling in the cartoon is aligned with the center of one of the regions of downwelling (negative vertical velocity) in the observations. The three-dimensional velocity field shown in (a)-(c) contains the features expected if this structure is drifting past the fixed instrument ( $P$ in the cartoon) with the observed sense of the crosswind mean flow ( $V$ in the cartoon). In particular, vertical downwelling is accompanied by positive downwind fluctuation velocity and a switch in sign of the crosswind fluctuation velocity from positive to negative. 
Documentation of similar events at this deeper ( $27 \mathrm{~m}$ vs. $15 \mathrm{~m}$ ) site suggest that this phenomenon may be more widespread than previously thought, and may be important to shelfwide particle transport. Additional support for this extended hypothesis comes from a SeaWiFs product that maps in-water particulates. The left panel of Figure 2 shows high land-derived particulate load nearshore and rapid decrease acrossshelf, a typical distribution of particulate material across the Georgia shelf. Only four days later (Figure 2, right), during passage of Tropical Storm Barry, the entire shelf exhibits high suspended particulate content. The LSC event (not shown) documented on this day was, therefore, part of a massive, shelfwide event. Effects of the quasi-organized structures of LSCs are not included in eddy diffusivity parameterizations of present numerical models of shallow seas, and their absence may account for the failure of such models to reproduce observed levels of cross-shelf flows (Edwards et al., 2006). Furthermore, because high sediment permeability requires the removal of fine particles that would tend to clog sediment interstices, LSC transport from the mid-shelf may also play a role in maintaining high permeability and advective exchange, which supports the high metabolic rates that characterize these sediments (Jahnke et al., 2005).

The seabed response to storm events is being examined using the BBL instrument packages and bed-imaging sonar. The initial results presented here focus on benthic fluxes of chlorophyll (Chl-a) estimated from fluorescence, which we assume to be associated predominantly with benthic diatoms. Figure 3 illustrates results from a storm in September 2007. Northeasterly winds developed on Day 258, attaining their maximum velocity (approx. $15 \mathrm{~m} \mathrm{~s}^{-1}$ ) on Day 259. Increased surface waves and associated near-bed oscillatory motions (Figure 3e) led to mobilization of the sand bed and development of distinct ripples on the bed surface (Figure 3f). Although prior to the storm Chl- $a$ was uniform in the near-bed layers (Figure 3a), during periods of high wave energy Chl- $a$ increased toward the bed (Figure 3b, c, d). Nearbed Chl- $a$ gradients at these times suggest that the seabed is the source of elevated Chl- $a$ in the BBL, and that the use of a vertical diffusion equation for representing flux from the bed is justified.

It is expected that ripple development
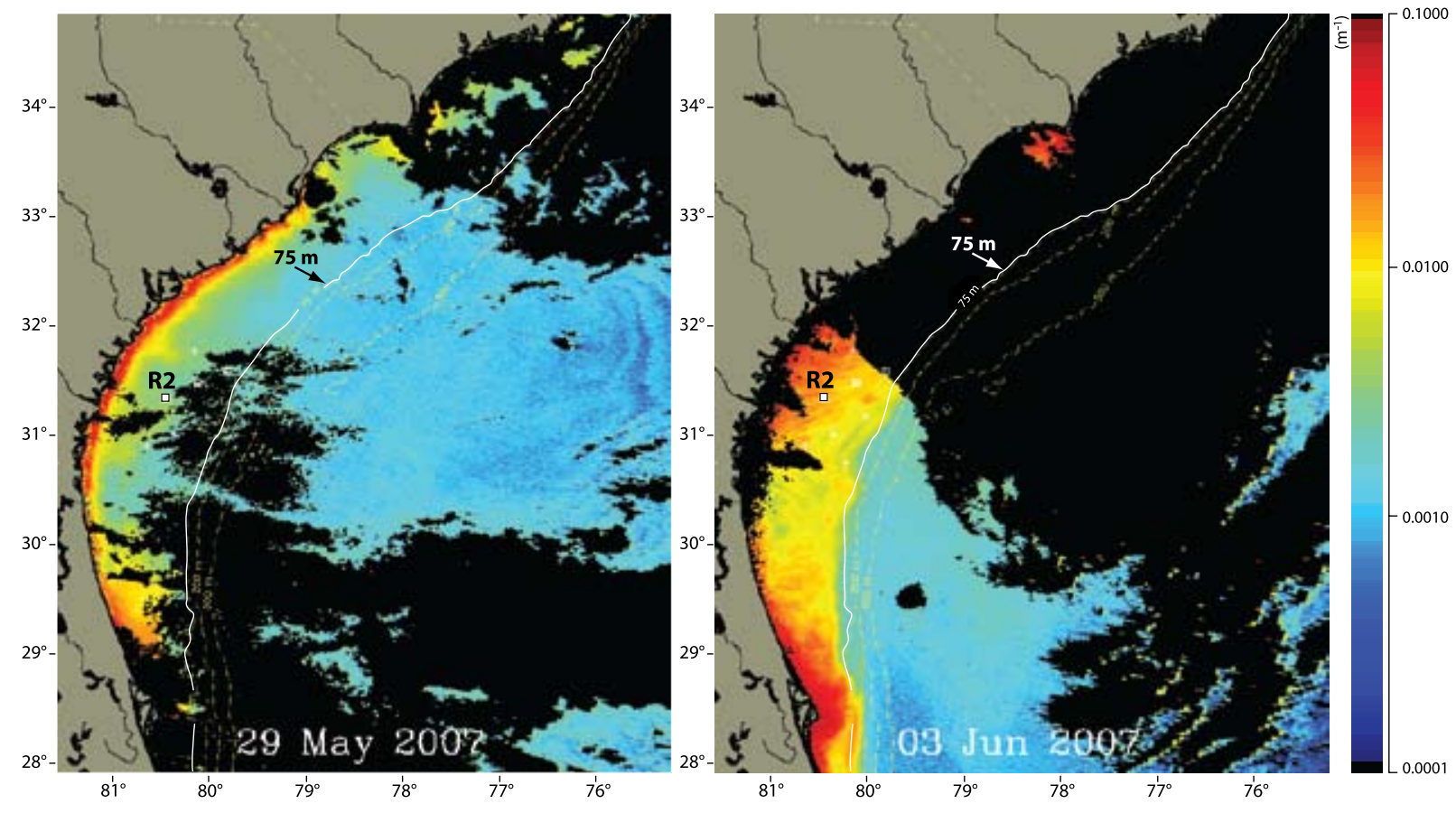

Figure 2. Maps of the SeaWiFS particulate backscatter (at $443 \mathrm{~nm}$ ) in the South Atlantic Bight before (left) and during (right) an LSC event observed at R2. The simultaneous occurrence of storm-induced resuspension and LSCs suggest that the latter could be a major contributor to cross-shelf particle transport. Black areas offshore are cloud covered. 

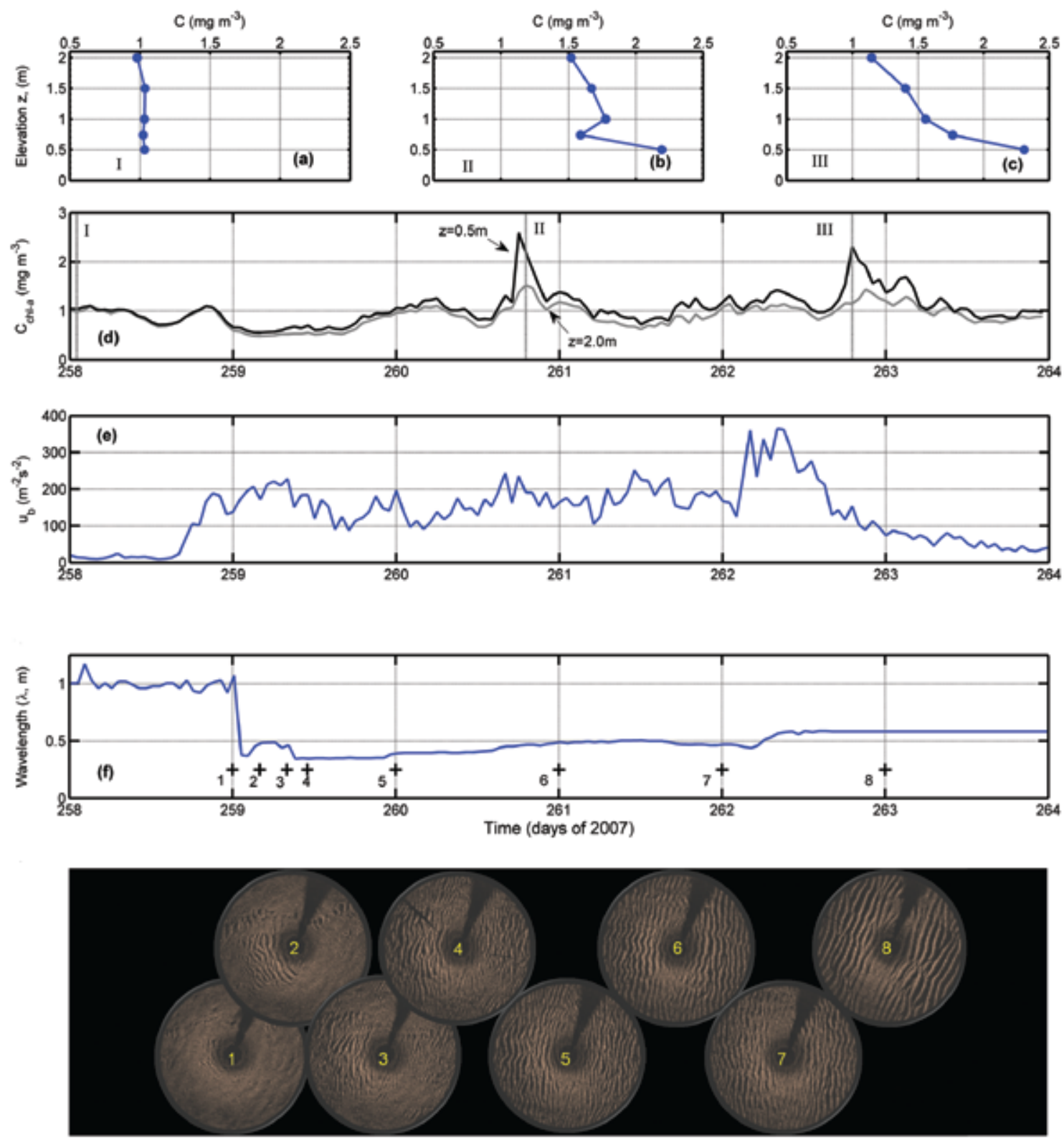

Figure 3. Results from benthic boundary layer instrument packages and the bed sonar from a storm event that occurred September 16-22, 2007 (Days 258 to 264). (a) to (c) Chl-a concentrations between 0.5-2.0 $\mathrm{m}$ above the seabed before (I) and during (II and III) the event. (d) Time-series of mean Chl-a concentration from 0.5-m and 2.0-m elevations above the seabed, with times of samples in (a)-(c) indicated. (e) Variance of wave orbital velocities. (f) Ripple wavelength extracted from the sonar images using an automated method (Voulgaris and Morin, 2008). Below (f) is a set of sonar images of the seabed obtained at the times indicated by crosses in ( $f$ ).

will affect vertical particle fluxes both directly and indirectly. Prior to the storm event, the seabed was almost flat (sonar image 1). At the onset of the storm, there was significant reorganization of the bed, with development of patches of ripples and transient variation of ripple length (Figure 3f). It is worth noting that near-bed Chl- $a$ began to exhibit a small vertical gradient (Figure $3 \mathrm{~d}$ ) only when the bed had developed ripples with a wavelength of about $40 \mathrm{~cm}$ (Figure $3 \mathrm{f}$, sonar images $2,3,4)$. The major peaks in near-bed Chl- $a$ (Figure 3d) occurred once the ripple field consisted of welldeveloped linear ripples. Although the exact relationship is the focus of further investigation, it is hypothesized that 
vortex shedding induced by the ripples may contribute to the pulsed flux of Chl- $a$ from the bed. Also, the rapid changes in bottom morphology (on time scales of hours), coupled with variations in wave height and seafloor currents (not shown), imply large variations in solute fluxes between porewaters of the permeable sand bed and the BBL. Once nearbed concentrations are elevated, then processes such as LSCs will be important for the vertical advection of particles and solutes into the water column.

These initial benthic observatory results document substantial variability in seafloor response to BBL forcing. Although discussions of sediment transport have long been framed in terms of episodic events, with controlling particle motion and resuspension thresholds, particle transfer to the seabed and porewater-bottom water solute exchange have generally not been discussed in this dynamic framework. Seabed morphology can change significantly over relatively short time scales (hours), altering seafloor-flow interactions and particle and solute exchange across the benthic interface. In addition, identification and quantification of aperiodic flows such as those associated with LSCs require continuous, high-resolution observations employing instrumentation with relatively large power requirements and high data-acquisition rates. Such measurements cannot practically be performed with battery-powered recording instrumentation. Recently, it has also been reported that ship-based light-flux profiles provide a biased assessment of long-term benthic light levels because operations cannot be performed across the full spectrum of sea states (Jahnke et al., 2008). Much of what happens on the seafloor occurs when researchers and their ship-based instruments are not or cannot be there. Without a sustained observational presence on the seafloor, many of the episodic events that may strongly influence the seabed and its interaction with the overlying water column will be missed. Robust benthic observatories will be required to generate a complete understanding of the statistics of near-bed forcing and the physical, chemical, and biological responses of the seafloor.

\section{ACKNOWLEDGEMENTS}

The authors thank the captain and crew of R/V Savannah, research divers Trent Moore and Mary Richards, and research coordinators Charles Robertson, Jeffrey Morin, and Julie Amft for the field effort that made these results possible. Research was supported by OCE0536326 (WS, AG, RJ, JN, and DS), OCE-053589 (GV), and OCE-0536345 (RS). 迦

\section{REFERENCES}

Bell, R.J., R.T. Short, F.H.W. Van Amerom, and R.H. Byrne. 2007. Calibration of an in situ membrane inlet mass spectrometer for measurements of dissolved gases and volatile organics in seawater. Environmental Science and Technology 41:8,123-8,128.

Edwards, K.P., J.A. Hare, F.E. Werner, and B.O. Blanton. 2006. Lagrangian circulation on the southeast US continental shelf: Implications for larval dispersal and retention. Continental Shelf Research 26:1,375-1,394.

Emery, K.O. 1968. Relict sediments on continental shelves of the world. American Association of Petroleum Geologists Bulletin 52:445-464.

Gargett, A., J. Wells, A.E. Tejada-Martinez, and C.E. Grosch. 2004. Langmuir supercells: A mechanism for sediment resuspension and transport in shallow seas. Science 306:1,925-1,928.

Huettel, M., and A. Rusch. 2000. Transport and degradation of phytoplankton in permeable sediment. Limnology and Oceanography 45:534-549.

Huettel, M., W. Ziebis, and S. Forster. 1996. Flowinduced uptake of particulate matter in permeable sediments. Limnology and Oceanography
41:309-322.

Jahnke, R., M. Richards, J. Nelson, C. Robertson, A. Rao, and D. Jahnke. 2005. Organic matter remineralization and porewater exchange rates in permeable South Atlantic Bight continental shelf sediments. Continental Shelf Research 25:1,433-1,452.

Jahnke, R.A., J.R. Nelson, M.E. Richards, C.Y. Robertson, A.M.F. Rao, and D. Jahnke. 2008. Benthic primary production on the Georgia midcontinental shelf: Benthic flux measurements and high-resolution, continuous in situ PAR records. Journal of Geophysical Research-Oceans 113(C8), doi:10.1029/2008JC004745.

Short, R.T., D.P. Fries, M.L. Kerr, C.E. Lembke, S.K. Toler, P.G. Wenner, and R.H. Byrne. 2001. Underwater mass spectrometers for in situ chemical analysis of the hydrosphere. Journal of the American Society for Mass Spectrometry 12:676-682.

Voulgaris, G., J.H. Trowbridge, J.J. Shaw, and A. Williams. 1998. High resolution measurements of turbulent fluxes and dissipation rates in the benthic boundary layer. Pp. 117-186 in Coastal Dynamics 97. E.B. Thornton, ed., American Society of Civil Engineers, Reston, VA.

Voulgaris, G., and J.P. Morin. 2008. A long-term real time sea bed morphology evolution system in the South Atlantic Bight. Pp. 71-79 in Proceedings of the IEEE/OES/CMTC Ninth Working Conference on Current Measurement Technology, 17-19 March, 2008. Charleston, SC. 